\title{
Moment convergence rates in the law of iterated logarithm for moving average process under dependence
}

\author{
Yayun Zhang and Qunying Wu*
}

\section{"Correspondence:} wqy666@glut.edu.cn College of Science, Guilin University of Technology, Guilin, 541006, P.R. China

\begin{abstract}
We assume that $X_{k}=\sum_{i=-\infty}^{+\infty} a_{i} \xi_{i+k}$ is a moving average process and $\left\{\xi_{i,}-\infty<i<+\infty\right\}$ is a doubly infinite sequence of identically distributed and dependent random variables with zero mean and finite variance and $\left\{a_{i},-\infty<i<+\infty\right\}$ is an absolutely summable sequence of real numbers. Under suitable conditions of dependence, we get the precise rates in the law of iterated logarithm for the first moment of the partial sums of the moving average process.
\end{abstract}

MSC: $60 \mathrm{~F} 15$

Keywords: precise asymptotics; the law of iterated logarithm; moment convergence; moving average; $\varphi$-mixing

\section{Introduction}

Suppose that $\left\{\xi_{i},-\infty<i<+\infty\right\}$ is a doubly infinite sequence of identically distributed random variables with zero mean and finite variance and $\left\{a_{i},-\infty<i<+\infty\right\}$ is an absolutely summable sequence of real numbers and

$$
X_{k}=\sum_{i=-\infty}^{+\infty} a_{i} \xi_{i+k}, \quad k \geq 1,
$$

is a moving average process based on $\left\{\xi_{i},-\infty<i<+\infty\right\}$. Set $S_{n}=\sum_{k=1}^{n} X_{k}, n \geq 1$.

Under the assumption that $\left\{\xi_{i},-\infty<i<+\infty\right\}$ is a sequence of independent identically distributed (i.i.d.) random variables, many limiting results have been obtained for the moving average process $\left\{X_{k}, k \geq 1\right\}$. Burton and Dehling [1] got a large deviation principle; Yang [2] established the central limit theorem and the law of the iterated logarithm; Li et al. [3] and Zhang [4] obtained the complete convergence; and complete moment convergence was proved by $\mathrm{Li}$ [5] and Li and Zhang [6].

In this article we will discuss the case of $\varphi$-mixing. Suppose that $\left\{\xi_{i},-\infty<i<+\infty\right\}$ is a sequence of identically distributed and $\varphi$-mixing random variables with

$$
\varphi(m):=\sup _{k \geq 1}\left\{|\mathbb{P}(B \mid A)-\mathbb{P}(B)|, A \in \mathcal{F}_{-\infty}^{k}, \mathbb{P}(A) \neq 0, B \in \mathcal{F}_{k+m}^{\infty}\right\} \rightarrow 0, \quad m \rightarrow \infty,
$$

(c) 2016 Zhang and Wu. This article is distributed under the terms of the Creative Commons Attribution 4.0 International License (http://creativecommons.org/licenses/by/4.0/), which permits unrestricted use, distribution, and reproduction in any medium, provided you give appropriate credit to the original author(s) and the source, provide a link to the Creative Commons license, and indicate if changes were made. 
where $\mathcal{F}_{a}^{b}=\sigma\left(\xi_{i}, a \leq i \leq b\right)$. By definition, we know that $\left\{X_{k}, k \geq 1\right\}$ is $\varphi$-mixing if $\left\{\xi_{i},-\infty<\right.$ $i<+\infty\}$ is a sequence of $\varphi$-mixing distributed random variables.

Since Hsu and Robbins [7] introduced complete convergence, there have been extensions in several directions. Some authors studied the precise asymptotics of complete convergence on moving average. For example, Li and Zhang [8] got precise asymptotics in the law of the iterated logarithm of moving average and Xiao and Yin [9] got moment convergence rates in the law of logarithm for moving average process under dependence, etc.

Xiao and Yin [10] studied precise asymptotics in the law of iterated logarithm for the first moment convergence of i.i.d. random variables. They got the following result.

Theorem 1.1 Let $\left\{Y, Y_{n}, n \geq 1\right\}$ be a sequence of i.i.d.random variables and set $U_{n}=$ $\sum_{i=1}^{n} Y_{i} . \mathcal{N}$ is the standard normal random variable and $\mathbb{E} Y=0, \mathbb{E} Y^{2}=\sigma^{2}$, then, for $d>0$ and $\beta>0$,

$$
\lim _{\varepsilon \searrow 0} \varepsilon^{2 \beta / d} \sum_{n=3}^{\infty} \frac{(\log \log n)^{\beta-1}}{n^{3 / 2} \log n} \mathbb{E}\left\{\left|U_{n}\right|-\varepsilon \sigma \sqrt{n}(\log \log n)^{d / 2}\right\}_{+}=\frac{d \sigma \mathbb{E}|\mathcal{N}|^{2 \beta / d+1}}{\beta(2 \beta+d)}
$$

Inspired by Xiao and Yin [10], we extend this result to moving average processes under $\varphi$-mixing random variable. Throughout this article, $\log x:=\ln (x \vee \mathrm{e})$, and the symbol $c$ denotes a positive constant which may be different in various places, and $[x]$ denotes the largest integer which is not greater than $x$, and $\mathcal{N}$ is the standard normal random variable. Now, we state the main result of this article.

Theorem 1.2 Suppose $\left\{X_{i}, i \geq 1\right\}$ is defined as in $(1)$, where $\left\{a_{i},-\infty<i<+\infty\right\}$ is a sequence of real numbers with $\sum_{i=-\infty}^{+\infty}\left|a_{i}\right|<\infty$, and $\left\{\xi_{i},-\infty<i<+\infty\right\}$ is a sequence of identically distributed $\varphi$-mixing random variables with zero mean and finite variance and $0<\sigma^{2}:=$ $\mathbb{E} \xi_{1}^{2}+2 \sum_{k=2}^{\infty} \mathbb{E} \xi_{1} \xi_{k}<\infty, \sum_{m=1}^{\infty} \varphi^{1 / 2}\left(2^{m}\right)<\infty, \tau:=\sigma\left|\sum_{i=-\infty}^{+\infty} a_{i}\right|$. Then, for $d>0$ and $\beta>0$,

$$
\lim _{\varepsilon \searrow 0} \varepsilon^{2 \beta / d} \sum_{n=3}^{\infty} \frac{(\log \log n)^{\beta-1}}{n^{3 / 2} \log n} \mathbb{E}\left\{\left|S_{n}\right|-\varepsilon \tau \sqrt{n}(\log \log n)^{d / 2}\right\}_{+}=\frac{d \tau \mathbb{E}|\mathcal{N}|^{2 \beta / d+1}}{\beta(2 \beta+d)}
$$

Remark 1.3 Let $a_{i}=1$ for $i=0$ and $a_{i}=0$ for $i \neq 0$, that is to say, $X_{k}=\xi_{k}$ with $\mathbb{E} \xi_{1}=$ $0, \mathbb{E} \xi_{1}^{2}<\infty$ and $0<\sigma^{2}:=\mathbb{E} \xi_{1}^{2}+2 \sum_{k=2}^{\infty} \mathbb{E} \xi_{1} \xi_{k}<\infty$, then, for $S_{n}=\sum_{k=1}^{n} \xi_{k}$, (3) still holds for $\tau=\sigma$ when $\left\{X_{k} ; k \geq 1\right\}$ is a sequence of identically distributed $\varphi$-mixing random variables. Therefore, this result extends Theorem 1.1.

\section{Lemmas}

To prove our main result, we need the following lemmas.

Lemma 2.1 (Burton and Dehling [1]) Let $\sum_{i=-\infty}^{+\infty} a_{i}$ be an absolutely convergent series of real numbers with $a=\sum_{i=-\infty}^{+\infty} a_{i}$ and $k \geq 1$, then

$$
\lim _{n \rightarrow \infty} \frac{1}{n} \sum_{i=-\infty}^{+\infty}\left|\sum_{j=i+1}^{i+n} a_{j}\right|^{k}=|a|^{k}
$$


Lemma 2.2 (Shao [11]) Let $\left\{X_{i}, i \geq 1\right\}$ be a sequence of $\varphi$-mixing random variables with zero means and finite second moments. Set $S_{n}=\sum_{i=1}^{n} X_{i}$. Then

$$
\mathbb{E} S_{n}^{2} \leq 8,000 \text { nexp }\left\{6 \sum_{i=1}^{[\log n]} \varphi^{1 / 2}\left(2^{i}\right)\right\} \max _{1 \leq i \leq n} \mathbb{E} X_{i}^{2} .
$$

If there is some $C_{n}$ such that $\max _{1 \leq i \leq n} \mathbb{E} S_{i}^{2} \leq C_{n}$, then, for all $q \geq 2$, there exists a $C=C(q, \varphi(\cdot))$ such that

$$
\mathbb{E} \max _{1 \leq i \leq n}\left|S_{i}\right|^{q} \leq C\left(C_{n}^{q / 2}+\mathbb{E} \max _{1 \leq i \leq n}\left|X_{i}\right|^{q}\right)
$$

Lemma 2.3 (Lin and Zhou [12]) Let $\left\{X_{i}, i \geq 1\right\}$ be defined as in (1), and $\left\{\xi_{i},-\infty<i<+\infty\right\}$ be a sequence of identically distributed $\varphi$-mixing random variables with zero means and finite variances and $0<\sigma^{2}=\mathbb{E} \xi_{1}^{2}+2 \sum_{k=2}^{\infty} \mathbb{E} \xi_{1} \xi_{k}<\infty, \sum_{m=1}^{\infty} \varphi^{1 / 2}\left(2^{m}\right)<\infty$, then

$$
\frac{S_{n}}{\tau \sqrt{n}} \stackrel{D}{\longrightarrow} \mathcal{N}(0,1), \quad \tau=\sigma\left|\sum_{i=-\infty}^{+\infty} a_{i}\right|,
$$

where $\stackrel{D}{\longrightarrow}$ denotes convergence in distribution.

\section{Proof of main result}

In this section, for $0<\varepsilon<1$ and $M>1$, we set

$$
b(\varepsilon)=\left[\exp \left\{\exp \left\{M \varepsilon^{-2 / d}\right\}\right\}\right]
$$

Without loss of generality, we assume that $\tau=1$.

Theorem 1.2 will be proved if we show the following propositions.

Proposition 3.1 Under the conditions of Theorem 1.2, we have

$$
\begin{aligned}
& \lim _{\varepsilon \searrow 0} \varepsilon^{2 \beta / d} \sum_{n=3}^{\infty} \frac{(\log \log n)^{\beta-1}}{n \log n} \mathbb{E}\left\{|\mathcal{N}|-\varepsilon(\log \log n)^{d / 2}\right\}_{+}=\frac{d \mathbb{E}|\mathcal{N}|^{2 \beta / d+1}}{\beta(2 \beta+d)}, \\
& \lim _{M \rightarrow \infty} \varepsilon^{2 \beta / d} \sum_{n>b(\varepsilon)} \frac{(\log \log n)^{\beta-1}}{n \log n} \mathbb{E}\left\{|\mathcal{N}|-\varepsilon(\log \log n)^{d / 2}\right\}_{+}=0
\end{aligned}
$$

where (9) uniformly holds true with respect to $0<\varepsilon<1$.

Proof See the proof of Proposition 1 and Proposition 3 in Xiao and Yin [10].

Proposition 3.2 Under the conditions of Theorem 1.2, we have

$$
\begin{gathered}
\lim _{\varepsilon \searrow 0} \varepsilon^{2 \beta / d} \sum_{n \leq b(\varepsilon)} \frac{(\log \log n)^{\beta-1}}{n \log n} \mid \mathbb{E}\left\{|\mathcal{N}|-\varepsilon(\log \log n)^{d / 2}\right\}_{+} \\
-\mathbb{E}\left\{\left|S_{n}\right| / \sqrt{n}-\varepsilon(\log \log n)^{d / 2}\right\}_{+} \mid=0 .
\end{gathered}
$$


Proof Let $\Delta_{n}=\sup _{x \in \mathbb{R}}\left|\mathbb{P}(|\mathcal{N}| \geq x)-\mathbb{P}\left(\left|S_{n}\right| / \sqrt{n} \geq x\right)\right|$. By Lemma 2.3, then we have $\Delta_{n} \rightarrow 0$ as $n \rightarrow \infty$. We have

$$
\begin{aligned}
& \varepsilon^{2 \beta / d} \sum_{n \leq b(\varepsilon)} \frac{(\log \log n)^{\beta-1}}{n \log n}\left|\mathbb{E}\left\{|\mathcal{N}|-\varepsilon(\log \log n)^{d / 2}\right\}_{+}-\mathbb{E}\left\{\left|S_{n}\right| / \sqrt{n}-\varepsilon(\log \log n)^{d / 2}\right\}_{+}\right| \\
& =\varepsilon^{2 \beta / d} \sum_{n \leq b(\varepsilon)} \frac{(\log \log n)^{\beta-1}}{n \log n} \mid \int_{0}^{\infty} \mathbb{P}\left(|\mathcal{N}| \geq x+\varepsilon(\log \log n)^{d / 2}\right) \mathrm{d} x \\
& -\int_{0}^{\infty} \mathbb{P}\left(\left|S_{n}\right| / \sqrt{n} \geq x+\varepsilon(\log \log n)^{d / 2}\right) \mathrm{d} x \mid \\
& \leq \varepsilon^{2 \beta / d} \sum_{n \leq b(\varepsilon)} \frac{(\log \log n)^{\beta-1+d / 2}}{n \log n} \int_{0}^{\infty} \mid \mathbb{P}\left(|\mathcal{N}| \geq(x+\varepsilon)(\log \log n)^{d / 2}\right) \\
& -\mathbb{P}\left(\left|S_{n}\right| / \sqrt{n} \geq(x+\varepsilon)(\log \log n)^{d / 2}\right) \mid \mathrm{d} x \\
& \leq \varepsilon^{2 \beta / d} \sum_{n \leq b(\varepsilon)} \frac{(\log \log n)^{\beta-1+d / 2}}{n \log n} \int_{0}^{l(n)} \mid \mathbb{P}\left(|\mathcal{N}| \geq(x+\varepsilon)(\log \log n)^{d / 2}\right) \\
& -\mathbb{P}\left(\left|S_{n}\right| / \sqrt{n} \geq(x+\varepsilon)(\log \log n)^{d / 2}\right) \mid \mathrm{d} x \\
& +\varepsilon^{2 \beta / d} \sum_{n \leq b(\varepsilon)} \frac{(\log \log n)^{\beta-1+d / 2}}{n \log n} \int_{l(n)}^{\infty} \mathbb{P}\left(|\mathcal{N}| \geq(x+\varepsilon)(\log \log n)^{d / 2}\right) \mathrm{d} x \\
& +\varepsilon^{2 \beta / d} \sum_{n \leq b(\varepsilon)} \frac{(\log \log n)^{\beta-1+d / 2}}{n \log n} \int_{l(n)}^{\infty} \mathbb{P}\left(\left|S_{n}\right| / \sqrt{n} \geq(x+\varepsilon)(\log \log n)^{d / 2}\right) \mathrm{d} x \\
& :=\mathrm{I}_{1}+\mathrm{I}_{2}+\mathrm{I}_{3} \text {, }
\end{aligned}
$$

where $l(n)=(\log \log n)^{-d / 2} \Delta_{n}^{-1 / 2}$. Hence, for $\mathrm{I}_{1}$, by applying Lemma 2.3 , we have

$$
\begin{aligned}
\mathrm{I}_{1} & \leq c \varepsilon^{2 \beta / d} \sum_{n \leq b(\varepsilon)} \frac{(\log \log n)^{\beta-1+d / 2}}{n \log n} \int_{0}^{l(n)} \Delta_{n} \mathrm{~d} x \leq c \varepsilon^{2 \beta / d} \sum_{n \leq b(\varepsilon)} \frac{(\log \log n)^{\beta-1+d / 2}}{n \log n} \Delta_{n} l(n) \\
& =c \varepsilon^{2 \beta / d} \sum_{n \leq b(\varepsilon)} \frac{(\log \log n)^{\beta-1}}{n \log n} \Delta_{n}^{1 / 2} \leq \frac{c M^{\beta}}{(\log \log b(\varepsilon))^{\beta}} \sum_{n \leq b(\varepsilon)} \frac{(\log \log n)^{\beta-1}}{n \log n} \Delta_{n}^{1 / 2}
\end{aligned}
$$

So, by the Toeplitz lemma, we have

$$
\lim _{\varepsilon \searrow 0} I_{1}=0
$$

As for $I_{2}$, by the Markov inequality, (12), and (13), we have

$$
\begin{aligned}
\mathrm{I}_{2} & \leq c \varepsilon^{2 \beta / d} \sum_{n \leq b(\varepsilon)} \frac{(\log \log n)^{\beta-1+d / 2}}{n \log n} \int_{l(n)}^{\infty} \frac{1}{(x+\varepsilon)^{2}(\log \log n)^{d}} \mathrm{~d} x \\
& \leq c \varepsilon^{2 \beta / d} \sum_{n \leq b(\varepsilon)} \frac{(\log \log n)^{\beta-1-d / 2}}{n \log n} l(n)^{-1} \\
& \leq c \varepsilon^{2 \beta / d} \sum_{n \leq b(\varepsilon)} \frac{(\log \log n)^{\beta-1}}{n \log n} \Delta_{n}^{1 / 2} \rightarrow 0, \quad \text { as } \varepsilon \searrow 0 .
\end{aligned}
$$


For $I_{3}$, note that $S_{n}=\sum_{k=1}^{n} X_{k}=\sum_{i=-\infty}^{+\infty} \sum_{k=1}^{n} a_{i} \xi_{i+k}=\sum_{i=-\infty}^{+\infty} a_{i} \sum_{j=i+1}^{i+n} \xi_{j}$. By Lemma 2.1, without loss of generality, we assume $\sum_{i=-\infty}^{+\infty}\left|a_{i}\right| \leq 1$, and set $S_{n}^{\prime}=\sum_{i=-\infty}^{+\infty} a_{i} \sum_{j=i+1}^{i+n} \xi_{j}^{\prime}$, where $\xi_{j}^{\prime}=\xi_{j} I\left(\left|\xi_{j}\right| \leq(x+\varepsilon) \sqrt{n}(\log \log n)^{d / 2}\right)$. By $\mathbb{E} \xi_{1}=0$, then, for all $x>0$, we have $\mathbb{E} \xi_{1} I\left(\left|\xi_{1}\right| \leq x\right)=-\mathbb{E} \xi_{1} I\left(\left|\xi_{1}\right|>x\right)$. So, we have

$$
\begin{aligned}
\left|\mathbb{E} S_{n}^{\prime}\right| & =\left|\sum_{i=-\infty}^{+\infty} a_{i} \mathbb{E} \sum_{j=i+1}^{i+n} \xi_{j} I\left(\left|\xi_{j}\right| \leq(x+\varepsilon) \sqrt{n}(\log \log n)^{d / 2}\right)\right| \\
& \leq \sum_{i=-\infty}^{+\infty}\left|a_{i}\right|\left|\mathbb{E} \sum_{j=i+1}^{i+n} \xi_{j} I\left(\left|\xi_{j}\right| \leq(x+\varepsilon) \sqrt{n}(\log \log n)^{d / 2}\right)\right| \\
& =\sum_{i=-\infty}^{+\infty}\left|a_{i}\right|\left|\mathbb{E} \sum_{j=i+1}^{i+n} \xi_{j} I\left(\left|\xi_{j}\right|>(x+\varepsilon) \sqrt{n}(\log \log n)^{d / 2}\right)\right| \\
& \leq n \mathbb{E}\left|\xi_{1}\right| I\left(\left|\xi_{1}\right|>(x+\varepsilon) \sqrt{n}(\log \log n)^{d / 2}\right) \\
& \leq \frac{\sqrt{n} \mathbb{E} \xi_{1}^{2}}{(x+\varepsilon)(\log \log n)^{d / 2}}
\end{aligned}
$$

thus for $x \geq l(n)$ and $n$ large enough, we have

$$
\frac{\left|\mathbb{E} S_{n}^{\prime}\right|}{(x+\varepsilon) \sqrt{n}(\log \log n)^{d / 2}} \leq \frac{c \mathbb{E} \xi_{1}^{2}}{(x+\varepsilon)^{2}(\log \log n)^{d}} \leq \frac{c}{l(n)^{2}(\log \log n)^{d}} \leq c \Delta_{n}<\frac{1}{4},
$$

so we obtain

$$
\begin{aligned}
& \int_{l(n)}^{\infty} \mathbb{P}\left(\left|S_{n}\right| / \sqrt{n} \geq(x+\varepsilon)(\log \log n)^{d / 2}\right) \mathrm{d} x \\
& \leq c \int_{l(n)}^{\infty} \mathbb{P}\left(\left|\sum_{i=-\infty}^{+\infty} a_{i} \sum_{j=i+1}^{i+n} \xi_{j} I\left(\left|\xi_{j}\right|>(x+\varepsilon) \sqrt{n}(\log \log n)^{d / 2}\right)\right|\right. \\
& \left.\quad>\frac{(x+\varepsilon) \sqrt{n}(\log \log n)^{d / 2}}{2}\right) \mathrm{d} x \\
& \quad+c \int_{l(n)}^{\infty} \mathbb{P}\left(\left|\sum_{i=-\infty}^{+\infty} a_{i} \sum_{j=i+1}^{i+n}\left(\xi_{j}^{\prime}-\mathbb{E} \xi_{j}^{\prime}\right)\right|>\frac{(x+\varepsilon) \sqrt{n}(\log \log n)^{d / 2}}{4}\right) \mathrm{d} x \\
& :=\mathrm{I}_{31}+\mathrm{I}_{32} .
\end{aligned}
$$

By (11) and (17), we have

$$
\mathrm{I}_{3} \leq c \varepsilon^{2 \beta / d} \sum_{n \leq b(\varepsilon)} \frac{(\log \log n)^{\beta-1+d / 2}}{n \log n}\left(\mathrm{I}_{31}+\mathrm{I}_{32}\right) .
$$

On the one hand, by (12) and (13), we obtain

$$
\begin{aligned}
& \varepsilon^{2 \beta / d} \sum_{n \leq b(\varepsilon)} \frac{(\log \log n)^{\beta-1+d / 2}}{n \log n} \mathrm{I}_{31} \\
& \quad \leq c \varepsilon^{2 \beta / d} \sum_{n \leq b(\varepsilon)} \frac{(\log \log n)^{\beta-1+d / 2}}{n \log n} \int_{l(n)}^{\infty} \frac{n \mathbb{E}\left|\xi_{1}\right| I\left(\left|\xi_{1}\right|>(x+\varepsilon) \sqrt{n}(\log \log n)^{d / 2}\right)}{\sqrt{n}(x+\varepsilon)(\log \log n)^{d / 2}} \mathrm{~d} x
\end{aligned}
$$




$$
\begin{aligned}
& \leq c \varepsilon^{2 \beta / d} \sum_{n \leq b(\varepsilon)} \frac{(\log \log n)^{\beta-1+d / 2}}{n \log n} \int_{l(n)}^{\infty} \frac{\mathbb{E} \xi_{1}^{2} I\left(\left|\xi_{1}\right|>(x+\varepsilon) \sqrt{n}(\log \log n)^{d / 2}\right)}{(x+\varepsilon)^{2}(\log \log n)^{d}} \mathrm{~d} x \\
& \leq c \varepsilon^{2 \beta / d} \sum_{n \leq b(\varepsilon)} \frac{(\log \log n)^{\beta-1-d / 2}}{n \log n} \int_{l(n)}^{\infty}(x+\varepsilon)^{-2} \mathrm{~d} x \\
& \leq c \varepsilon^{2 \beta / d} \sum_{n \leq b(\varepsilon)} \frac{(\log \log n)^{\beta-1-d / 2}}{n \log n} l(n)^{-1} \\
& \leq c \varepsilon^{2 \beta / d} \sum_{n \leq b(\varepsilon)} \frac{(\log \log n)^{\beta-1}}{n \log n} \Delta_{n}^{1 / 2} \rightarrow 0, \quad \text { as } \varepsilon \searrow 0 .
\end{aligned}
$$

On the other hand, by the Markov inequality, the Hölder inequality, and Lemma 2.2, and noting that $\sum_{m=1}^{\infty} \varphi^{1 / 2}\left(2^{m}\right)<\infty$, we have

$$
\begin{aligned}
& \varepsilon^{2 \beta / d} \sum_{n \leq b(\varepsilon)} \frac{(\log \log n)^{\beta-1+d / 2}}{n \log n} \mathrm{I}_{32} \\
& \leq c \varepsilon^{2 \beta / d} \sum_{n \leq b(\varepsilon)} \frac{(\log \log n)^{\beta-1+d / 2-d q / 2}}{n^{1+q / 2} \log n} \int_{l(n)}^{\infty}(x+\varepsilon)^{-q} \mathbb{E}\left|\sum_{i=-\infty}^{+\infty} a_{i} \sum_{j=i+1}^{i+n}\left(\xi_{j}^{\prime}-\mathbb{E} \xi_{j}^{\prime}\right)\right|^{q} \mathrm{~d} x \\
& \leq c \varepsilon^{2 \beta / d} \sum_{n \leq b(\varepsilon)} \frac{(\log \log n)^{\beta-1+d / 2-d q / 2}}{n^{1+q / 2} \log n} \\
& \times \int_{l(n)}^{\infty}(x+\varepsilon)^{-q} \mathbb{E}\left[\sum_{i=-\infty}^{+\infty}\left(\left|a_{i}\right|^{1-\frac{1}{q}}\right)\left(\left|a_{i}\right|^{\frac{1}{q}}\left|\sum_{j=i+1}^{i+n}\left(\xi_{j}^{\prime}-\mathbb{E} \xi_{j}^{\prime}\right)\right|\right)\right]^{q} \mathrm{~d} x \\
& \leq c \varepsilon^{2 \beta / d} \sum_{n \leq b(\varepsilon)} \frac{(\log \log n)^{\beta-1+d / 2-d q / 2}}{n^{1+q / 2} \log n} \\
& \times \int_{l(n)}^{\infty}(x+\varepsilon)^{-q} \mathbb{E}\left[\left(\sum_{i=-\infty}^{+\infty}\left|a_{i}\right|^{1-\frac{1}{q} \cdot \frac{1}{1-\frac{1}{q}}}\right)^{1-\frac{1}{q}}\left(\sum_{i=-\infty}^{+\infty}\left|a_{i}\right|^{\frac{1}{q} \cdot q}\left|\sum_{j=i+1}^{i+n}\left(\xi_{j}^{\prime}-\mathbb{E} \xi_{j}^{\prime}\right)\right|^{q}\right)^{\frac{1}{q}}\right]^{q} \mathrm{~d} x \\
& \leq c \varepsilon^{2 \beta / d} \sum_{n \leq b(\varepsilon)} \frac{(\log \log n)^{\beta-1+d / 2-d q / 2}}{n^{1+q / 2} \log n} \\
& \times \int_{l(n)}^{\infty}(x+\varepsilon)^{-q} \mathbb{E}\left(\sum_{i=-\infty}^{+\infty}\left|a_{i}\right|\right)^{\left(1-\frac{1}{q}\right) q} \sum_{i=-\infty}^{+\infty}\left|a_{i}\right|\left|\sum_{j=i+1}^{i+n}\left(\xi_{j}^{\prime}-\mathbb{E} \xi_{j}^{\prime}\right)\right|^{q} \mathrm{~d} x \\
& \leq c \varepsilon^{2 \beta / d} \sum_{n \leq b(\varepsilon)} \frac{(\log \log n)^{\beta-1+d / 2-d q / 2}}{n^{1+q / 2} \log n} \\
& \times \int_{l(n)}^{\infty}(x+\varepsilon)^{-q} \sum_{i=-\infty}^{+\infty}\left|a_{i}\right| \mathbb{E}\left|\sum_{j=i+1}^{i+n}\left(\xi_{j}^{\prime}-\mathbb{E} \xi_{j}^{\prime}\right)\right|^{q} \mathrm{~d} x \\
& \leq c \varepsilon^{2 \beta / d} \sum_{n \leq b(\varepsilon)} \frac{(\log \log n)^{\beta-1+d / 2-d q / 2}}{n^{1+q / 2} \log n} \int_{l(n)}^{\infty}(x+\varepsilon)^{-q}\left(\left(n \mathbb{E} \xi_{1}^{\prime 2}\right)^{\frac{q}{2}}+n \mathbb{E}\left|\xi_{1}^{\prime}\right|^{q}\right) \mathrm{d} x \\
& :=\mathrm{I}_{321}+\mathrm{I}_{322} \text {. }
\end{aligned}
$$


For $\mathrm{I}_{321}, q \geq 2$. By (12) and (13), we have

$$
\begin{aligned}
\mathrm{I}_{321} & \leq c \varepsilon^{2 \beta / d} \sum_{n \leq b(\varepsilon)} \frac{(\log \log n)^{\beta-1+d / 2-d q / 2}}{n \log n} \int_{l(n)}^{\infty}(x+\varepsilon)^{-q} \mathrm{~d} x \\
& \leq c \varepsilon^{2 \beta / d} \sum_{n \leq b(\varepsilon)} \frac{(\log \log n)^{\beta-1+d / 2-d q / 2}}{n \log n} l(n)^{1-q} \\
& \leq c \varepsilon^{2 \beta / d} \sum_{n \leq b(\varepsilon)} \frac{(\log \log n)^{\beta-1}}{n \log n} \Delta_{n}^{\frac{q-1}{2}} \rightarrow 0, \quad \text { as } \varepsilon \searrow 0 .
\end{aligned}
$$

For $\mathrm{I}_{322}$, by (12) and (13), we have

$$
\begin{aligned}
\mathrm{I}_{322} \leq & c \varepsilon^{2 \beta / d} \sum_{n \leq b(\varepsilon)} \frac{(\log \log n)^{\beta-1+d / 2-d q / 2}}{n^{q / 2} \log n} \\
& \times \int_{l(n)}^{\infty}(x+\varepsilon)^{-q} \mathbb{E}\left|\xi_{1}\right|^{q} I\left(\left|\xi_{1}\right| \leq(x+\varepsilon) \sqrt{n}(\log \log n)^{d / 2}\right) \mathrm{d} x \\
\leq & c \varepsilon^{2 \beta / d} \sum_{n \leq b(\varepsilon)} \frac{(\log \log n)^{\beta-1-d / 2}}{n \log n} \\
& \times \int_{l(n)}^{\infty}(x+\varepsilon)^{-2} \mathbb{E} \xi_{1}^{2} I\left(\left|\xi_{1}\right| \leq(x+\varepsilon) \sqrt{n}(\log \log n)^{d / 2}\right) \mathrm{d} x \\
\leq & c \varepsilon^{2 \beta / d} \sum_{n \leq b(\varepsilon)} \frac{(\log \log n)^{\beta-1-d / 2}}{n \log n} l(n)^{-1} \\
= & c \varepsilon^{2 \beta / d} \sum_{n \leq b(\varepsilon)} \frac{(\log \log n)^{\beta-1}}{n \log n} \Delta_{n}^{1 / 2} \rightarrow 0, \quad \text { as } \varepsilon \searrow 0 .
\end{aligned}
$$

Thus, combining with (13), (14), and (18)-(20), we complete the proof of this proposition.

Proposition 3.3 Under the conditions of Theorem 1.2,

$$
\lim _{M \rightarrow \infty} \varepsilon^{2 \beta / d} \sum_{n>b(\varepsilon)} \frac{(\log \log n)^{\beta-1}}{n \log n} \mathbb{E}\left\{\left|S_{n}\right| / \sqrt{n}-\varepsilon(\log \log n)^{d / 2}\right\}_{+}=0
$$

uniformly holds true with respect to $0<\varepsilon<1$.

Proof For $x \geq 0, M$ large enough, we have

$$
\frac{\left|\mathbb{E} S_{n}^{\prime}\right|}{\sqrt{n}(x+\varepsilon)(\log \log n)^{d / 2}} \leq \frac{c}{(x+\varepsilon)^{2}(\log \log n)^{d}} \leq \frac{c}{M^{d}}<\frac{1}{4} .
$$

Then

$$
\begin{aligned}
& \mathbb{P}\left(\left|S_{n}\right| \geq \sqrt{n}(\log \log n)^{d / 2}(x+\varepsilon)\right) \\
& \quad \leq \mathbb{P}\left(\left|\sum_{i=-\infty}^{+\infty} a_{i} \sum_{j=i+1}^{i+n} \xi_{j} I\left(\left|\xi_{j}\right|>(x+\varepsilon) \sqrt{n}(\log \log n)^{d / 2}\right)\right|>\frac{\sqrt{n}(\log \log n)^{d / 2}(x+\varepsilon)}{2}\right)
\end{aligned}
$$




$$
\begin{aligned}
& +\mathbb{P}\left(\left|S_{n}^{\prime}\right|>\frac{\sqrt{n}(\log \log n)^{d / 2}(x+\varepsilon)}{2}\right) \\
\leq & \mathbb{P}\left(\left|\sum_{i=-\infty}^{+\infty} a_{i} \sum_{j=i+1}^{i+n} \xi_{j} I\left(\left|\xi_{j}\right|>(x+\varepsilon) \sqrt{n}(\log \log n)^{d / 2}\right)\right|>\frac{\sqrt{n}(\log \log n)^{d / 2}(x+\varepsilon)}{2}\right) \\
& +\mathbb{P}\left(\left|S_{n}^{\prime}-\mathbb{E} S_{n}^{\prime}\right| \geq\left|S_{n}^{\prime}\right|-\left|\mathbb{E} S_{n}^{\prime}\right|\right. \\
& \left.>\frac{\sqrt{n}(\log \log n)^{d / 2}(x+\varepsilon)}{2}-\frac{\sqrt{n}(\log \log n)^{d / 2}(x+\varepsilon)}{4}\right) \\
= & \mathbb{P}\left(\left|\sum_{i=-\infty}^{+\infty} a_{i} \sum_{j=i+1}^{i+n} \xi_{j} I\left(\left|\xi_{j}\right|>(x+\varepsilon) \sqrt{n}(\log \log n)^{d / 2}\right)\right|>\frac{\sqrt{n}(\log \log n)^{d / 2}(x+\varepsilon)}{2}\right) \\
& +\mathbb{P}\left(\left|\sum_{i=-\infty}^{+\infty} a_{i} \sum_{j=i+1}^{i+n}\left(\xi_{j}^{\prime}-\mathbb{E} \xi_{j}^{\prime}\right)\right|>\frac{\sqrt{n}(\log \log n)^{d / 2}(x+\varepsilon)}{4}\right) .
\end{aligned}
$$

Therefore, by (22), we obtain

$$
\begin{aligned}
\sum_{n>b(\varepsilon)} & \frac{(\log \log n)^{\beta-1}}{n \log n} \mathbb{E}\left\{\left|S_{n}\right| / \sqrt{n}-\varepsilon(\log \log n)^{\frac{d}{2}}\right\}_{+} \\
= & \sum_{n>b(\varepsilon)} \frac{(\log \log n)^{\beta-1}}{n \log n} \int_{0}^{\infty} \mathbb{P}\left(\left|S_{n}\right| / \sqrt{n} \geq x+\varepsilon(\log \log n)^{d / 2}\right) \mathrm{d} x \\
= & \sum_{n>b(\varepsilon)} \frac{(\log \log n)^{\beta-1+\frac{d}{2}}}{n \log n} \int_{0}^{\infty} \mathbb{P}\left(\left|S_{n}\right| \geq \sqrt{n}(\log \log n)^{\frac{d}{2}}(x+\varepsilon)\right) \mathrm{d} x \quad\left(x=y(\log \log n)^{d / 2}\right) \\
\leq & \sum_{n>b(\varepsilon)} \frac{(\log \log n)^{\beta-1+\frac{d}{2}}}{n \log n} \int_{0}^{\infty} \mathbb{P}\left(\mid \sum_{i=-\infty}^{+\infty} a_{i} \sum_{j=i+1}^{i+n} \xi_{j} I\left(\left|\xi_{j}\right|\right.\right. \\
& \left.\left.>(x+\varepsilon) \sqrt{n}(\log \log n)^{\frac{d}{2}}\right) \mid>\frac{(x+\varepsilon) \sqrt{n}(\log \log n)^{\frac{d}{2}}}{2}\right) \mathrm{d} x \\
& +\sum_{n>b(\varepsilon)} \frac{(\log \log n)^{\beta-1+\frac{d}{2}}}{n \log n} \int_{0}^{\infty} \mathbb{P}\left(\left|\sum_{i=-\infty}^{+\infty} a_{i} \sum_{j=i+1}^{i+n}\left(\xi_{j}^{\prime}-\mathbb{E} \xi_{j}^{\prime}\right)\right|\right. \\
& \left.>\frac{(x+\varepsilon) \sqrt{n}(\log \log n)^{\frac{d}{2}}}{4}\right) \mathrm{d} x \\
:= & J_{1}+J_{2} .
\end{aligned}
$$

For $J_{1}$, when $2 \beta<d$, we have

$$
\begin{aligned}
\varepsilon^{2 \beta / d} J_{1} & \leq c \varepsilon^{2 \beta / d} \sum_{n>b(\varepsilon)} \frac{(\log \log n)^{\beta-1+d / 2}}{n \log n} \int_{0}^{\infty} \frac{n \mathbb{E}\left|\xi_{1}\right| I\left(\left|\xi_{1}\right|>(x+\varepsilon) \sqrt{n}(\log \log n)^{d / 2}\right)}{(x+\varepsilon) \sqrt{n}(\log \log n)^{d / 2}} \mathrm{~d} x \\
& \leq c \varepsilon^{2 \beta / d} \sum_{n>b(\varepsilon)} \frac{(\log \log n)^{\beta-1-d / 2}}{n \log n} \int_{0}^{\infty} \frac{\mathbb{E} \xi_{1}^{2} I\left(\left|\xi_{1}\right|>(x+\varepsilon) \sqrt{n}(\log \log n)^{d / 2}\right)}{(x+\varepsilon)^{2}} \mathrm{~d} x \\
& \leq c \varepsilon^{2 \beta / d} \sum_{n>b(\varepsilon)} \frac{(\log \log n)^{\beta-1-d / 2}}{n \log n} \int_{0}^{\infty}(x+\varepsilon)^{-2} \mathrm{~d} x
\end{aligned}
$$




$$
\begin{aligned}
& \leq c \varepsilon^{2 \beta / d-1} \sum_{n>b(\varepsilon)} \frac{(\log \log n)^{\beta-1-d / 2}}{n \log n} \\
& \leq c M^{\beta-d / 2}
\end{aligned}
$$

thus

$$
\lim _{M \rightarrow \infty} \varepsilon^{2 \beta / d} J_{1}=0
$$

uniformly holds true with respect to $0<\varepsilon<1$.

When $2 \beta \geq d$, we have

$$
\begin{aligned}
J_{1} \leq & c \sum_{n>b(\varepsilon)} \frac{(\log \log n)^{\beta-1+d / 2}}{n \log n} \int_{0}^{\infty} \frac{n \mathbb{E}\left|\xi_{1}\right| I\left(\left|\xi_{1}\right|>(x+\varepsilon) \sqrt{n}(\log \log n)^{d / 2}\right)}{(x+\varepsilon) \sqrt{n}(\log \log n)^{d / 2}} \mathrm{~d} x \\
= & c \sum_{n>b(\varepsilon)} \frac{(\log \log n)^{\beta-1}}{\sqrt{n} \log n} \int_{0}^{\infty} \frac{\mathbb{E}\left|\xi_{1}\right| I\left(\left|\xi_{1}\right|>(x+\varepsilon) \sqrt{n}(\log \log n)^{d / 2}\right)}{x+\varepsilon} \mathrm{d} x \\
= & c \int_{0}^{\infty} \frac{1}{x+\varepsilon} \sum_{n>b(\varepsilon)} \frac{(\log \log n)^{\beta-1}}{\sqrt{n} \log n} \sum_{j=n}^{\infty} \mathbb{E}\left|\xi_{1}\right| I\left(\sqrt{j}(\log \log j)^{d / 2}<\frac{\left|\xi_{1}\right|}{x+\varepsilon}\right. \\
& \left.\leq \sqrt{j+1}(\log \log (j+1))^{d / 2}\right) \mathrm{d} x \\
= & c \int_{0}^{\infty} \frac{1}{x+\varepsilon} \sum_{j>b(\varepsilon)} \mathbb{E}\left|\xi_{1}\right| I\left(\sqrt{j}(\log \log j)^{\frac{d}{2}}<\frac{\left|\xi_{1}\right|}{x+\varepsilon} \leq \sqrt{j+1}(\log \log (j+1))^{\frac{d}{2}}\right) \\
& \times \sum_{n=b(\varepsilon)+1}^{j} \frac{(\log \log n)^{\beta-1}}{\sqrt{n} \log n} \mathrm{~d} x .
\end{aligned}
$$

Since we have $(\log \log n)^{\beta-1}(\log n)^{-1} \rightarrow 0$, as $n>b(\varepsilon) \rightarrow \infty$, then we obtain

$$
\begin{aligned}
J_{1} & \leq c \int_{0}^{\infty} \frac{1}{x+\varepsilon} \sum_{j>b(\varepsilon)} \sqrt{j} \mathbb{E}\left|\xi_{1}\right| I\left(\sqrt{j}(\log \log j)^{d / 2}<\frac{\left|\xi_{1}\right|}{x+\varepsilon} \leq \sqrt{j+1}(\log \log (j+1))^{d / 2}\right) \mathrm{d} x \\
& \leq c \int_{0}^{\infty} \frac{1}{(x+\varepsilon)^{2}} \mathbb{E} \xi_{1}^{2} I\left(\left|\xi_{1}\right|>(x+\varepsilon) \sqrt{b(\varepsilon)}(\log \log b(\varepsilon))^{d / 2}\right) \mathrm{d} x \\
& \leq c \int_{0}^{\infty} \frac{1}{(x+\varepsilon)^{2}} \mathbb{E} \xi_{1}^{2} I\left(\left|\xi_{1}\right|>\varepsilon(\log \log b(\varepsilon))^{d / 2}\right) \mathrm{d} x \\
& =c \varepsilon^{-1} \mathbb{E} \xi_{1}^{2} I\left(\left|\xi_{1}\right|>\varepsilon(\log \log b(\varepsilon))^{d / 2}=M^{d / 2}\right) .
\end{aligned}
$$

Hence, when $2 \beta \geq d$,

$$
\lim _{M \rightarrow \infty} \varepsilon^{2 \beta / d} J_{1} \leq \lim _{M \rightarrow \infty} \mathbb{E} \xi_{1}^{2} I\left(\left|\xi_{1}\right|>M^{d / 2}\right)=0
$$

uniformly holds true with respect to $0<\varepsilon<1$. 
Next we consider $J_{2}$, similar to the proof of $I_{32}$, we have

$$
\begin{aligned}
\varepsilon^{2 \beta / d} J_{2} & \leq c \varepsilon^{2 \beta / d} \sum_{n>b(\varepsilon)} \frac{(\log \log n)^{\beta-1+\frac{d}{2}-\frac{d q}{2}}}{n^{1+\frac{q}{2}} \log n} \int_{0}^{\infty}(x+\varepsilon)^{-q}\left(\left(n \mathbb{E} \xi_{1}^{\prime 2}\right)^{q / 2}+n \mathbb{E}\left|\xi_{1}^{\prime}\right|^{q}\right) \mathrm{d} x \\
& :=J_{21}+J_{22} .
\end{aligned}
$$

For $J_{21}$, haven taken $q>\max \{2 \beta / d+1,2\}$, then we have

$$
\begin{aligned}
J_{21} & \leq c \varepsilon^{2 \beta / d} \sum_{n>b(\varepsilon)} \frac{(\log \log n)^{\beta-1+\frac{d}{2}-\frac{d q}{2}}}{n \log n} \int_{0}^{\infty}(x+\varepsilon)^{-q} \mathrm{~d} x \\
& \leq c \varepsilon^{2 \beta / d-q+1} \sum_{n>b(\varepsilon)} \frac{(\log \log n)^{\beta-1+\frac{d}{2}-\frac{d q}{2}}}{n \log n} \\
& \leq c \varepsilon^{2 \beta / d-q+1}(\log \log b(\varepsilon))^{\beta+\frac{d}{2}-\frac{d q}{2}} \\
& =M^{\beta+\frac{d}{2}-\frac{d q}{2}}
\end{aligned}
$$

Thus

$$
\lim _{M \rightarrow \infty} J_{21}=0
$$

uniformly holds true with respect to $0<\varepsilon<1$.

For $J_{22}$, if $2 \beta<d$, we have

$$
\begin{aligned}
J_{22} \leq & c \varepsilon^{2 \beta / d} \sum_{n>b(\varepsilon)} \frac{(\log \log n)^{\beta-1+\frac{d}{2}-\frac{d q}{2}}}{n^{\frac{q}{2}} \log n} \\
& \times \int_{0}^{\infty}(x+\varepsilon)^{-q} \mathbb{E}\left|\xi_{1}\right|^{q} I\left(\left|\xi_{1}\right| \leq(x+\varepsilon) \sqrt{n}(\log \log n)^{d / 2}\right) \mathrm{d} x \\
\leq & c \varepsilon^{2 \beta / d} \sum_{n>b(\varepsilon)} \frac{(\log \log n)^{\beta-1-\frac{d}{2}}}{n \log n} \\
& \times \int_{0}^{\infty}(x+\varepsilon)^{-2} \mathbb{E} \xi_{1}^{2} I\left(\left|\xi_{1}\right| \leq(x+\varepsilon) \sqrt{n}(\log \log n)^{d / 2}\right) \mathrm{d} x \\
\leq & c \varepsilon^{2 \beta / d} \sum_{n>b(\varepsilon)} \frac{(\log \log n)^{\beta-1-\frac{d}{2}}}{n \log n} \int_{0}^{\infty}(x+\varepsilon)^{-2} \mathrm{~d} x \\
\leq & c \varepsilon^{2 \beta / d-1} \sum_{n>b(\varepsilon)} \frac{(\log \log n)^{\beta-1-\frac{d}{2}}}{n \log n} \\
= & c M^{\beta-d / 2} .
\end{aligned}
$$

Thus

$$
\lim _{M \rightarrow \infty} J_{22}=0
$$

uniformly holds true with respect to $0<\varepsilon<1$. 
If $2 \beta \geq d$, we divide $J_{22}$ into two parts:

$$
\begin{aligned}
J_{22} \leq & c \varepsilon^{2 \beta / d} \sum_{n>b(\varepsilon)} \frac{(\log \log n)^{\beta-1+\frac{d}{2}-\frac{d q}{2}}}{n^{\frac{q}{2}} \log n} \\
& \times \int_{0}^{\infty}(x+\varepsilon)^{-q} \mathbb{E}\left|\xi_{1}\right|^{q} I\left(\left|\xi_{1}\right| \leq(x+\varepsilon) \sqrt{n}(\log \log n)^{d / 2}\right) \mathrm{d} x \\
\leq & c \varepsilon^{2 \beta / d} \sum_{n>b(\varepsilon)} \frac{(\log \log n)^{\beta-1+\frac{d}{2}-\frac{d q}{2}}}{n^{\frac{q}{2}} \log n} \int_{0}^{\infty}(x+\varepsilon)^{-q} \mathbb{E}\left|\xi_{1}\right|^{q}\left[I\left(\left|\xi_{1}\right| \leq \sqrt{n}(\log \log n)^{d / 2} \varepsilon\right)\right. \\
& \left.+I\left(\sqrt{n}(\log \log n)^{d / 2} \varepsilon<\left|\xi_{1}\right| \leq(x+\varepsilon) \sqrt{n}(\log \log n)^{d / 2}\right)\right] \mathrm{d} x \\
:= & J_{221}+J_{222 .} .
\end{aligned}
$$

For $J_{221}$, since $q>2$, so we have

$$
\begin{aligned}
J_{221} \leq & c \varepsilon^{2 \beta / d-q+1} \sum_{n>b(\varepsilon)} \frac{(\log \log n)^{\beta-1+\frac{d}{2}-\frac{d q}{2}}}{n^{\frac{q}{2}} \log n} \mathbb{E}\left|\xi_{1}\right|^{q} I\left(\left|\xi_{1}\right| \leq \sqrt{b(\varepsilon)}(\log \log b(\varepsilon))^{\frac{d}{2}} \varepsilon\right) \\
& +c \varepsilon^{2 \beta / d-q+1} \sum_{n>b(\varepsilon)} \frac{(\log \log n)^{\beta-1+\frac{d}{2}-\frac{d q}{2}}}{n^{\frac{q}{2}} \log n} \\
& \times \sum_{j=b(\varepsilon)+1}^{n} \mathbb{E}\left|\xi_{1}\right|^{q} I\left(\sqrt{j-1}(\log \log (j-1))^{\frac{d}{2}} \varepsilon<\left|\xi_{1}\right| \leq \sqrt{j}(\log \log j)^{\frac{d}{2}} \varepsilon\right) \\
\leq & c \varepsilon^{2 \beta / d-1} \frac{(\log \log b(\varepsilon))^{\beta-1-\frac{d}{2}}}{\log b(\varepsilon)} \mathbb{E} \xi_{1}^{2} I\left(\left|\xi_{1}\right| \leq \sqrt{b(\varepsilon)}(\log \log b(\varepsilon))^{\frac{d}{2}} \varepsilon\right) \\
& +c \varepsilon^{2 \beta / d-q+1} \sum_{j=b(\varepsilon)+1}^{\infty} \mathbb{E}\left|\xi_{1}\right|^{q} I\left(\sqrt{j-1}(\log \log (j-1))^{\frac{d}{2}} \varepsilon<\left|\xi_{1}\right| \leq \sqrt{j}(\log \log j)^{\frac{d}{2}} \varepsilon\right) \\
& \times \sum_{n=j}^{\infty} \frac{(\log \log n)^{\beta-1+\frac{d}{2}-\frac{d q}{2}}}{n^{\frac{q}{2}} \log n} \\
\leq & c \varepsilon^{2 \beta / d-1} \frac{1}{\log \log b(\varepsilon)} \\
& +c \varepsilon^{2 \beta / d-q+1} \sum_{j=b(\varepsilon)+1}^{\infty} \mathbb{E}\left|\xi_{1}\right|^{q} I\left(\sqrt{j-1}(\log \log (j-1))^{\frac{d}{2}} \varepsilon<\left|\xi_{1}\right| \leq \sqrt{j}(\log \log j)^{\frac{d}{2}} \varepsilon\right) j^{1-\frac{q}{2}} \\
\leq & c M^{-1}+c E^{2 \beta / d-1+d / 2} M^{2} I\left(\left|\xi_{1}\right|>\sqrt{b(\varepsilon)}(\log \log b(\varepsilon))^{\frac{d}{2}} \varepsilon>M^{\frac{d}{2}}\right) \\
& \times \mathbb{E} \xi_{1}^{2} I\left(\sqrt{j-1}(\log \log (j-1))^{\frac{d}{2}} \varepsilon<\left|\xi_{1}\right| \leq \sqrt{j}(\log \log j)^{\frac{d}{2}} \varepsilon\right) \\
& \times \frac{(\log \log j)^{\beta-1+\frac{d}{2}-\frac{d q}{2}}}{\log j} \sum_{j=b(\varepsilon)+1}^{\infty} \frac{(\log \log j)^{\beta-1-\frac{d}{2}}}{\log j} \\
& \\
&
\end{aligned}
$$


Thus

$$
\lim _{M \rightarrow \infty} J_{221} \leq \lim _{M \rightarrow \infty} M^{-1}+\lim _{M \rightarrow \infty} \mathbb{E} \xi_{1}^{2} I\left(\left|\xi_{1}\right|>M^{\frac{d}{2}}\right)=0
$$

uniformly holds true with respect to $0<\varepsilon<1$.

Finally we consider $J_{222}$, since $2 \beta \geq d$ and $q>2$, we have

$$
\begin{aligned}
J_{222} \leq & c \varepsilon^{2 \beta / d} \sum_{n>b(\varepsilon)} \frac{(\log \log n)^{\beta-1+\frac{d}{2}-\frac{d q}{2}}}{n^{\frac{q}{2}} \log n} \mathbb{E}\left|\xi_{1}\right|^{q} I\left(\left|\xi_{1}\right|>\sqrt{n}(\log \log n)^{\frac{d}{2}} \varepsilon\right) \\
& \times \int_{0}^{\infty}(x+\varepsilon)^{-q} I\left(\left|\xi_{1}\right| \leq(x+\varepsilon) \sqrt{n}(\log \log n)^{\frac{d}{2}}\right) \mathrm{d} x \\
\leq & c \varepsilon^{2 \beta / d} \sum_{n>b(\varepsilon)} \frac{(\log \log n)^{\beta-1+\frac{d}{2}-\frac{d q}{2}}}{n^{\frac{q}{2}} \log n} \mathbb{E}\left|\xi_{1}\right|^{q} I\left(\left|\xi_{1}\right|>\sqrt{n}(\log \log n)^{\frac{d}{2}} \varepsilon\right) \\
& \times \int_{\frac{\left|\xi_{1}\right|}{\sqrt{n}(\log \log n)^{\frac{d}{2}}-\varepsilon}}^{\infty}(x+\varepsilon)^{-q} \mathrm{~d} x \\
\leq & c \varepsilon^{2 \beta / d} \sum_{n>b(\varepsilon)} \frac{(\log \log n)^{\beta-1}}{\sqrt{n} \log n} \mathbb{E}\left|\xi_{1}\right| I\left(\left|\xi_{1}\right|>\sqrt{n}(\log \log n)^{\frac{d}{2}} \varepsilon\right) \\
\leq & c \varepsilon^{2 \beta / d} \sum_{n>b(\varepsilon)} \frac{(\log \log n)^{\beta-1}}{\sqrt{n} \log n} \\
& \times \sum_{j=n}^{\infty} \mathbb{E}\left|\xi_{1}\right| I\left(\sqrt{j}(\log \log j)^{\frac{d}{2}} \varepsilon<\left|\xi_{1}\right| \leq \sqrt{j+1}(\log \log (j+1))^{\frac{d}{2}} \varepsilon\right) \\
\leq & c \varepsilon^{2 \beta / d} \sum_{j=b(\varepsilon)}^{\infty} \mathbb{E}\left|\xi_{1}\right| I\left(\sqrt{j}(\log \log j)^{\frac{d}{2}} \varepsilon<\left|\xi_{1}\right| \leq \sqrt{j+1}(\log \log (j+1))^{\frac{d}{2}} \varepsilon\right) \\
\leq & c \varepsilon^{2 \beta / d-1} \sum_{j=b(\varepsilon)}^{\infty} \mathbb{E} \xi_{1}^{2} I\left(\sqrt{j}(\log \log j)^{\frac{d}{2}} \varepsilon<\left|\xi_{1}\right| \leq \sqrt{j+1}(\log \log (j+1))^{\frac{d}{2}} \varepsilon\right) \\
\leq & c \varepsilon^{2 \beta / d-1} \mathbb{E} \xi_{1}^{2} I\left(\left|\xi_{1}\right| \geq \varepsilon \sqrt{b(\varepsilon)}(\log \log b(\varepsilon))^{\frac{d}{2}}>M^{\frac{d}{2}}\right) \\
& \times \sum_{n=b(\varepsilon)}^{j} \frac{(\log \log n)^{\beta-1}}{\sqrt{n} \log n} \\
& \sqrt{j} \mathbb{E}\left|\xi_{1}\right| I\left(\sqrt{j}(\log \log j)^{\frac{d}{2}} \varepsilon<\left|\xi_{1}\right| \leq \sqrt{j+1}(\log \log (j+1))^{\frac{d}{2}} \varepsilon\right) \\
&
\end{aligned}
$$

Hence,

$$
\lim _{M \rightarrow \infty} J_{222} \leq \lim _{M \rightarrow \infty} \varepsilon^{2 \beta / d-1} \mathbb{E} \xi_{1}^{2} I\left(\left|\xi_{1}\right|>M^{d / 2}\right) \leq \lim _{M \rightarrow \infty} \mathbb{E} \xi_{1}^{2} I\left(\left|\xi_{1}\right|>M^{d / 2}\right)=0
$$

uniformly holds true with respect to $0<\varepsilon<1$.

Thus, combining with (23)-(28), we complete the proof of this proposition. 
Combining with Proposition 3.1, Proposition 3.2, and Proposition 3.3 we complete the proof of Theorem 1.2.

\section{Competing interests}

The authors declare that they have no competing interests.

\section{Authors' contributions}

All authors contributed equally to the writing of this paper. All authors read and approved the final manuscript.

\section{Authors' information}

Qunying Wu is a professor, doctor, working in the field of probability and statistics.

\section{Acknowledgements}

The authors would like to thank the referees and the editors for the helpful comments and suggestions. This work was supported by the National Natural Science Foundation of China $(11361019,11661029)$ and the Guangxi China Science Foundation (2015GXNSFAA139008).

Received: 12 June 2016 Accepted: 28 September 2016 Published online: 18 October 2016

\section{References}

1. Burton, RM, Dehling, H: Large deviations for some weakly dependent random processes. Stat. Probab. Lett. 9, 397-401 (1990)

2. Yang, X-Y: The law of the iterated logarithm and stochastic index central limit theorem of B-valued stationary linear processes. Chin. Ann. Math., Ser. A 17, 703-714 (1996)

3. Li, D, Rao, M-B, Wang, X: Complete convergence of moving average processes. Stat. Probab. Lett. 14, 111-114 (1992)

4. Zhang, L-X: Complete convergence of moving average processes under dependence assumptions. Stat. Probab. Lett. 30, 165-170 (1996)

5. Li, Y-X: Precise asymptotics in complete moment convergence of moving-average processes. Stat. Probab. Lett. 76, $1305-1315(2006)$

6. Li, Y-X, Zhang, L-X: Complete moment convergence of moving-average processes under dependence assumptions. Stat. Probab. Lett. 70, 191-197 (2004)

7. Hsu, PL, Robbins, H: Complete convergence and the law of large numbers. Proc. Natl. Acad. Sci. USA 33, 25-31 (1947)

8. Li, Y-X, Zhang, L-X: Precise asymptotics in the law of the iterated logarithm of moving-average processes. Acta Math. Sin. 22, 143-156 (2006)

9. Xiao, $X-Y, Y i n, H-W$ : Moment convergence rates in the law of logarithm for moving average process under dependence. Stoch. Int. J. Probab. Stoch. Process. 86(1), 1-15 (2014)

10. Xiao, $X-Y$, Yin, $H-W$ : Precise asymptotics in the law of iterated logarithm for the first moment convergence of iid random variables. Stat. Probab. Lett. 82, 1590-1596 (2012)

11. Shao, Q-M: A moment inequality and its application. Acta Math. Sin. 31, 736-747 (1988)

12. Lin, Z-Y, Zhou, H: Precise asymptotics of complete moment convergence on moving average. Acta Math. Sin. Engl. Ser. 28(12), 2507-2526 (2012)

\section{Submit your manuscript to a SpringerOpen ${ }^{\ominus}$ journal and benefit from:}

- Convenient online submission

Rigorous peer review

- Immediate publication on acceptance

- Open access: articles freely available online

- High visibility within the field

- Retaining the copyright to your article 